\title{
Fabrication of a Bottom Electrode for a Nano-scale Beam Resonator Using Backside Exposure with a Self-aligned Metal Mask
}

\author{
Yong-Seok Lee*, Yun-Ho Jang*, Yong-Seung Bang*, Jung-Mu Kim**, \\ Jong-Man Kim*** and Yong-Kweon Kim ${ }^{\dagger}$
}

\begin{abstract}
In this paper, we describe a self-aligned fabrication method for a nano-patterned bottom electrode using flood exposure from the backside. Misalignments between layers could cause the final devices to fail after the fabrication of the nano-scale bottom electrodes. A self-alignment was exploited to embed the bottom electrode inside the glass substrate. Aluminum patterns act as a dry etching mask to fabricate glass trenches as well as a self-aligned photomask during the flood exposure from the backside. The patterned photoresist (PR) has a negative sidewall slope using the flood exposure. The sidewall slopes of the glass trench and the patterned PR were $54.00^{\circ}$ and $63.47^{\circ}$, respectively. The negative sidewall enables an embedment of a gold layer inside $0.7 \mu \mathrm{m}$ wide glass trenches. Gold residues on the trench edges were removed by the additional flood exposure with wet etching. The sidewall slopes of the patterned PR are related to the slopes of the glass trenches. Nano-scale bottom electrodes inside the glass trenches will be used in beam resonators operating at high resonant frequencies.
\end{abstract}

Keywords: Nano-scale vertical beam resonator, Self-aligned mask, Backside flood exposure

\section{Introduction}

Microelectromechanical systems (MEMS) resonators have been widely used in the applications of inertial measurement sensors, RF filers, mass sensors, etc. Recently, many research groups have tried to reduce the size of resonators up to a nano-scale as size reduction can offer a lot of advantages such as high speed operation, a low actuation force, a high integration level, and high sensitivity [1-3]. Beam resonators are one of the representative devices in nano-scale RF MEMS due to their simple fabrication and structural stability, and they can be classified into lateral and vertical resonators according to the vibration direction [4]. Vertical beam resonators have advantages over lateral beam resonators in view of their high integrity and easy fabrication in an array.

The realization of the nano-scale vertical beam resonator is still a challenge due to fabrication issues such as tight alignment margin, beam stiction, and so on. An accurate alignment between patterned layers, in particular, becomes more problematic as the device size becomes smaller up to nano-scale.

Generally, two photo masks are required to deposit the metal lines inside the glass trench as shown in Fig. 1. The first mask is for the etching to form the glass trench, and

+ Corresponding Author : Dept. of Electrical Engineering and Computer Science, Seoul National University, Korea (yongkkim@snu.ac.kr)

* Dept. of Electrical Engineering and Computer Science, Seoul National University, Korea

** Division of Electrical, Electronic and Computer Engineering, Chonbuk National University, Korea

*** Dept. of Nanosystem and Nanoprocess Engineering, Pusan Nantional University, Korea

Received : June 25, 2009; Accepted : September 3, 2009 the second is for the lift-off process to pattern the metal line inside the trench. In conventional photolithography, however, an alignment error is larger than tens of nanometer, meaning that exact alignment is impossible. Therefore, device failures such as an electrical short problem can occur due to misalignment between the photolithography processes of glass etching and metal patterning.

In this study we propose a fabrication method for nanoscale bottom electrodes inside glass trenches using a flood exposure from the backside which eliminates alignment errors. The glass substrate was pre-etched using the aluminum etching mask for the final release of the nano-scale beam resonator. Negative sloped photoresist (PR) formed by the self-aligned UV flood exposure from the backside enabled the embedment of gold electrodes inside the glass trenches using a lift-off process.

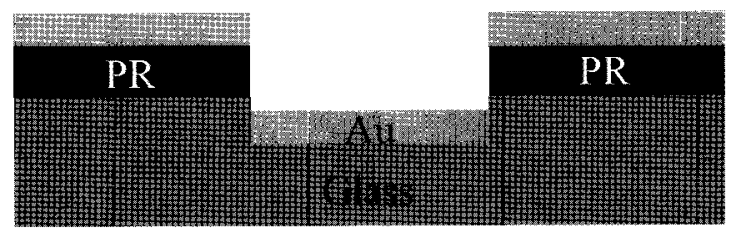

(a)

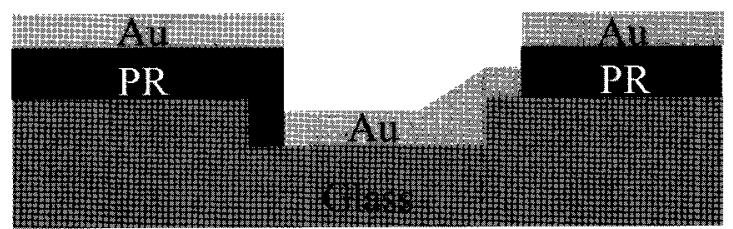

(b)

Fig. 1. Metal (gold) patterning inside the glass trench; (a) with accurate alignment, (b) with misalignment 


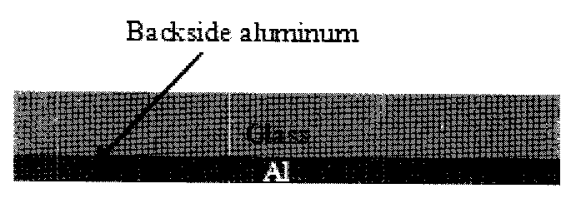

(a)

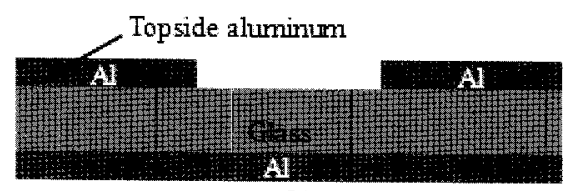

(b)

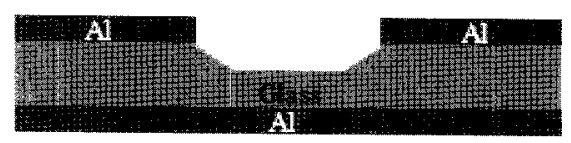

(c)

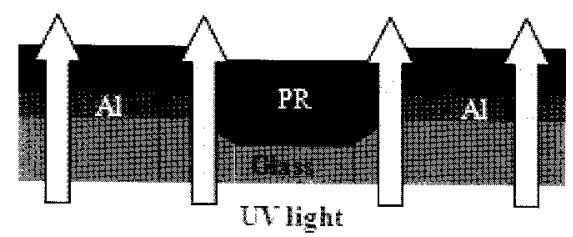

(d)

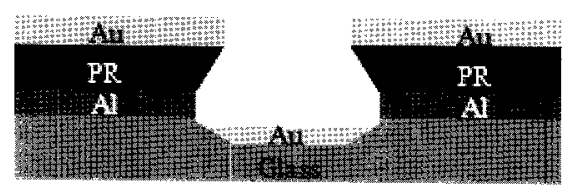

(e)

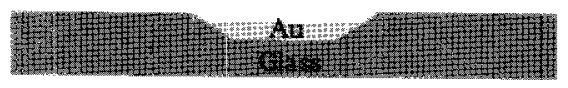

(f)

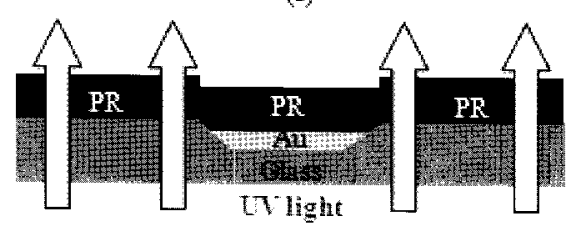

(g)

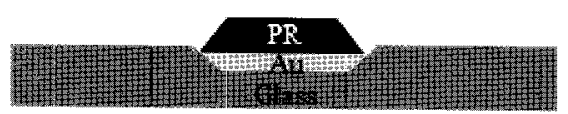

(h)

Fig. 2. Fabrication process: (a) aluminum evaporation on the backside of the wafer, (b) aluminum lift-off on the topside, (c) glass dry etch, (d) UV flood exposure from the backside using the self-aligned aluminum mask, (e) gold $0.2 \mu \mathrm{m}$ evaporation, (f) gold lift-off, (g) additional backside photolithography, (h) timed etching of gold residues.

Note that the self-aligned PR on top of the gold lines should be cured for 3 minutes at $110{ }^{\circ} \mathrm{C}$ to protect the gold lines during the gold wet etching stage

\section{Fabrication Method}

The fabrication process starts with aluminum evaporation on the backside of a glass wafer (Pyrex \#7740, Corning Co., Ltd.) to make an optically opaque substrate in the first photolithography (Fig. 1(a)). We obtained nano-scale patterns less than $0.7 \mu \mathrm{m}$ using a positive PR (TDMR AR87, Tokyo-Ohka Kogyo Co., Ltd.). A $0.2 \mu \mathrm{m}$ thick aluminum layer was evaporated on the topside of the glass wafer for a glass etching mask, where the aluminum layer should be thick enough to block the UV light in the following backside flood exposure. The deposited top side aluminum layer was patterned using a lift-off process by removing the underlaid TDMR AR-87 in acetone (Fig. 2(b)). The glass trenches were formed using two dry etch pieces of equipment, the Oxford RIE 80 and Precision-5000, to obtain different etching profiles (Fig. 2(c)). AZ 1512 was coated as a passivation layer on the topside when the backside aluminum layer was removed in a phosphoric-acetic-nitric (PAN) solution to make a transparent surface for the backside flood exposure. After removing the AZ 1512, TDMR AR-87 was coated on the topside to be exposed from the backside. The aluminum patterns on the topside utilized as a glass etching mask played the role of self-aligned photo mask during the backside UV flood exposure (Fig. 2(d)). The negative sidewall slopes of the TDMR AR-87 were obtained via backside UV flood exposure on the aluminum patterns (Fig. 2(e)). A $0.2 \mu \mathrm{m}$ thick gold layer was evaporated and bottom electrodes were patterned inside the glass trenches using a lift-off process (Fig. 2(f)). The patterned TDMR AR-87 on the topside was used as a sacrificial layer in the lift-off process, thus a well developed negative PR profile is desired [5]. Additional backside UV flood exposure was performed to remove the gold residues along the edges of the glass trenches, where gold patterns inside the glass trenches were used as a self-aligned photomask (Fig. $2(\mathrm{~g})$ ). Positive PR remained on top of the gold lines after exposure and development. Gold timed wet etching removes the residual gold layer, which improves electrical isolation (Fig. 2(h)).

\section{Results and Discussions}

The PR profile is usually determined by several conditions such as UV intensity, exposure time, substrate reflection, and so on. When exposure conditions are optimized, the PR profile is directly influenced by the UV light path [6]. As shown in Fig. 3, if light passes from the glass to the PR during the backside exposure, refraction occurs at the interface between the PR and glass layers.

The refraction at the interface determines the UV path in the PR, and it is also strongly related to the PR's profile. The slopes of the glass trenches depend on the etching conditions. Consequently, the glass trenches were etched using two different pieces of dry etch equipment, the Oxford RIE 80 and Precision-5000, to identify the relationship between sidewall slope angles of the glass trenches and the 


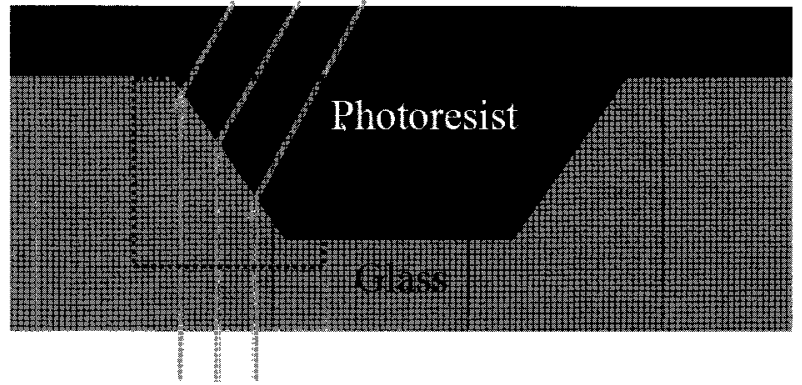

Fig. 3. UV light refraction at the interface between glass and PR

TDMR AR-87. The experimental conditions were summarized in Table 1 . The Oxford RIE 80 etcher was employed to obtain the anisotropic profile of the glass trenches using $\mathrm{SF}_{6}$ and $\mathrm{Ar}$ and the glass was etched for $180 \mathrm{sec}$ at 0.1 Torr. The Precision-5000 was used for the slanted profile using $\mathrm{CHF}_{3}, \mathrm{CF}_{4}$, and $\mathrm{Ar}$ for $100 \mathrm{sec}$ at 0.13 Torr to make the glass trenches.

The refraction at the interface determines the UV path in the PR, and it is also strongly related to the PR's profile. The slopes of the glass trenches depend on the etching conditions. Consequently, the glass trenches were etched using two different pieces of dry etch equipment, the Oxford RIE 80 and Precision-5000, to identify the relationship between sidewall slope angles of the glass trenches and the TDMR AR-87. The experimental conditions were summarized in Table 1. The Oxford RIE 80 etcher was employed to obtain the anisotropic profile of the glass trenches using $\mathrm{SF}_{6}$ and $\mathrm{Ar}$ and the glass was etched for $180 \mathrm{sec}$ at 0.1 Torr. The Precision-5000 was used for the slanted profile using $\mathrm{CHF}_{3}, \mathrm{CF}_{4}$, and $\mathrm{Ar}$ for $100 \mathrm{sec}$ at 0.13 Torr to make the glass trenches.

Figs. 4 and 5 show the cross sectional views of the $0.7 \mu \mathrm{m}$ wide glass trenches after glass etching and backside flood exposure using the Oxford RIE 80 and Precision5000 , respectively. The $0.2 \mu \mathrm{m}$ thick aluminum was patterned for the glass etching mask and the glass trenches were formed in a trapezoidal shape. The PR had slanted sidewall profiles similar to those of the glass trenches after backside UV flood exposure. More anisotropic etching was performed using the Oxford RIE 80 than the Precision5000 . The surface roughness of the glass trenches was better with the Precision-5000 than with the Oxford RIE 80, and it was reflected in the sidewall profiles of the TDMR AR-87.

Table 1. Glass dry etching conditions

\begin{tabular}{c|c|c}
\hline & Oxford RIE 80 & Precision-5000 \\
\hline \multirow{3}{*}{ Gas flow rate } & $\begin{array}{c}\mathrm{SF}_{6}: 100 \mathrm{sccm} \\
\mathrm{Ar}: 50 \mathrm{sccm}\end{array}$ & $\begin{array}{c}\mathrm{CHF}_{3}: 25 \mathrm{sccm} \\
\mathrm{CF}_{4}: 5 \mathrm{sccm} \\
\text { Ar: } 50 \mathrm{sccm}\end{array}$ \\
\hline Pressure & 0.1 Torr & $0.13 \mathrm{Torr}$ \\
\hline Etch time & $180 \mathrm{sec}$ & $100 \mathrm{sec}$ \\
\hline
\end{tabular}

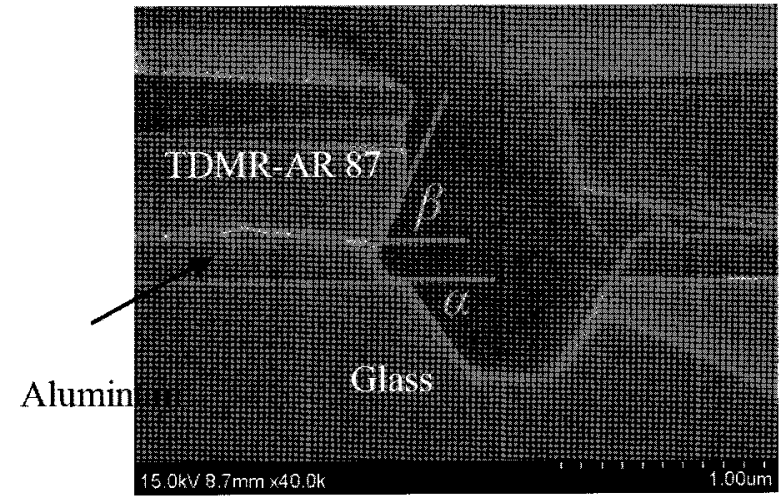

Fig. 4. Cross sectional view of a $0.7 \mu \mathrm{m}$ wide trench after backside photolithography where the glass was etched with the Precision-5000

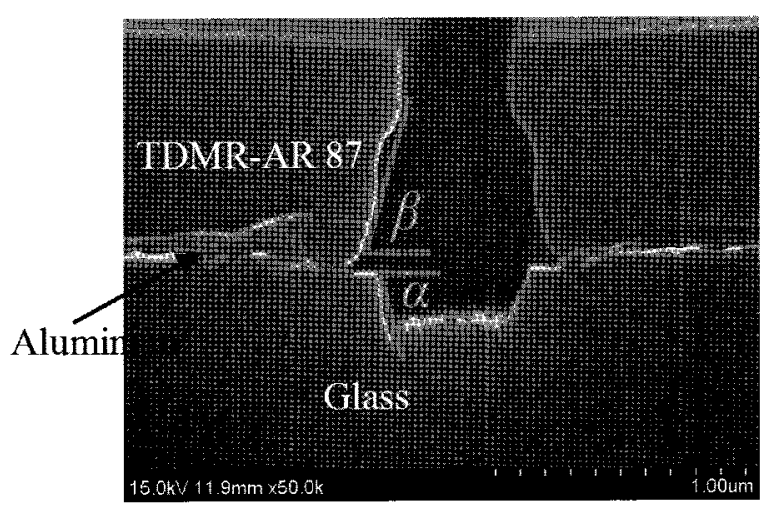

Fig. 5. Cross sectional view of a $0.7 \mu \mathrm{m}$ wide trench after backside photolithography where the glass was etched with the Oxford RIE 80

The sidewall angles of the glass trenches $(\alpha)$ and the TDMR AR-87 $(\beta)$ were measured to quantify the glass and PR profiles. The measured $\alpha$ and $\beta$ were summarized in Table 2 and plotted in Fig. 6.

The sidewall slope angle of the glass trenches $(\alpha)$ and the TDMR AR-87 $(\beta)$ were measured for 4 different linewidth samples, namely, $0.7,0.8,0.9$, and $1.0 \mu \mathrm{m}$, respectively. A relatively small angle of the glass trenches $(\alpha)$ as well as the angle of the TDMR AR-87 $(\beta)$ was observed when using the Precision-5000 compared with the results of the Oxford RIE 80. The sidewall slope angle of the glass trench was $54.00^{\circ}$ and the corresponding sidewall angle of the TDMR-AR 87 was $63.47^{\circ}$ for a $0.7 \mu \mathrm{m}$ wide glass

Table 2. Sidewall slopes of glass trenches and PR

\begin{tabular}{c|c|c|c|c}
\hline & \multicolumn{4}{|c}{ Sidewall slope } \\
\hline \multirow{2}{*}{$\begin{array}{c}\text { trench } \\
\text { width } \\
(\mu \mathrm{m})\end{array}$} & \multicolumn{2}{|c}{ Oxford RIE 80 } & \multicolumn{2}{c}{ Precision-5000 } \\
\cline { 2 - 5 } & $\alpha$ & $\beta$ & $\alpha$ & $\beta$ \\
\hline 0.7 & $69.42^{\circ}$ & $71.39^{\circ}$ & $54.00^{\circ}$ & $63.47^{\circ}$ \\
\hline 0.8 & $74.09^{\circ}$ & $73.34^{\circ}$ & $56.34^{\circ}$ & $67.76^{\circ}$ \\
\hline 0.9 & $75.89^{\circ}$ & $73.34^{\circ}$ & $57.01^{\circ}$ & $70.75^{\circ}$ \\
\hline 1.0 & $76.00^{\circ}$ & $78.73^{\circ}$ & $59.01^{\circ}$ & $70.75^{\circ}$ \\
\hline
\end{tabular}




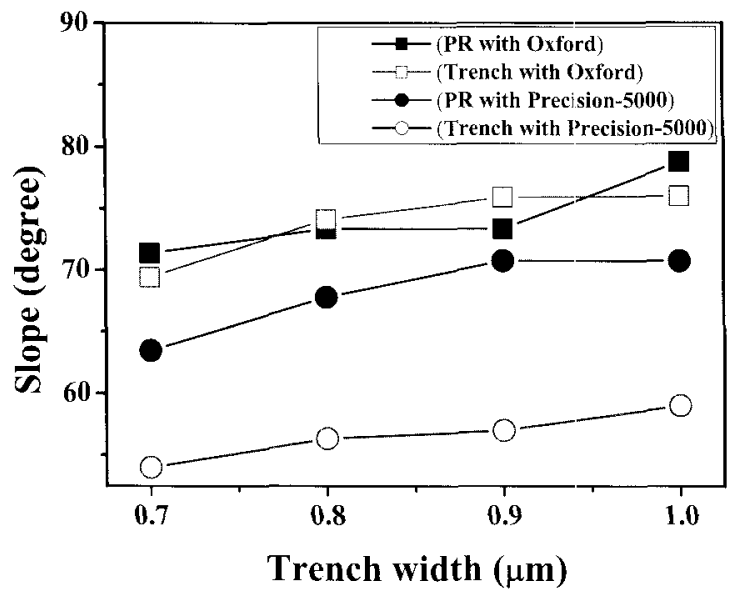

Fig. 6. Sidewall slopes of glass trenches and TDMR AR-87 with respect to trench widths

trench when the glass was etched using the Precision-5000.

The sidewall slope angle of the TDMR AR-87 $(\beta)$ increased as the slope angle of the glass trench $(\alpha)$ increased due to an optical refraction [7], [8].

The Precision-5000 was adapted to make a negative sloped profile of the TDMR-AR 87 and the $0.2 \mu \mathrm{m}$ thick gold layer was evaporated on the TDMR AR-87 after the backside UV flood exposure. After the lift-off process, the gold layer was well patterned inside the glass trenches as a bottom electrode (Fig. 7). An unwanted gold layer remained along the edge of the trench which seems to be the result of the directionality of the gold particles during evaporation. It was imperative to remove the gold residues for electrical isolation since the gold residues may cause an electrical short problem after the nano-scale beam resonator is formed over the glass trench.

The gold residues along the edge of the glass trench were removed using an additional backside UV flood ex-

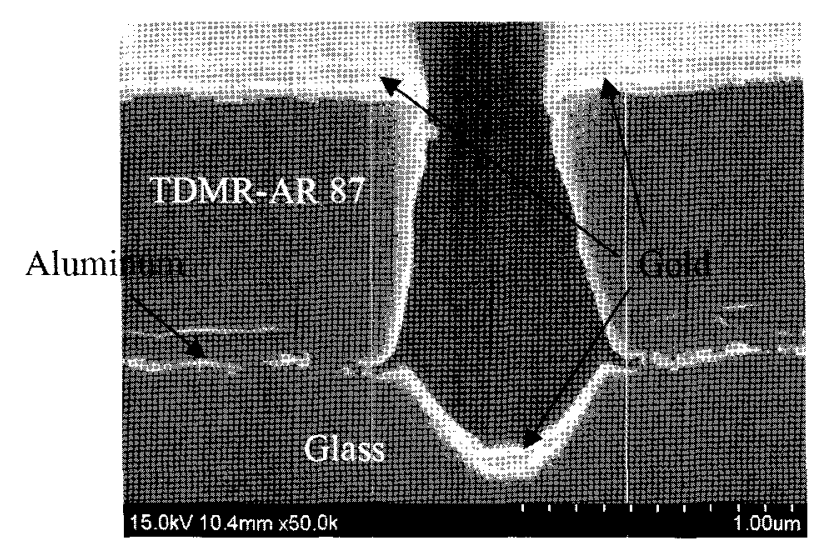

Fig. 7. Cross sectional view of the glass trench with a gold metal line and negative sloped PR after gold evaporation

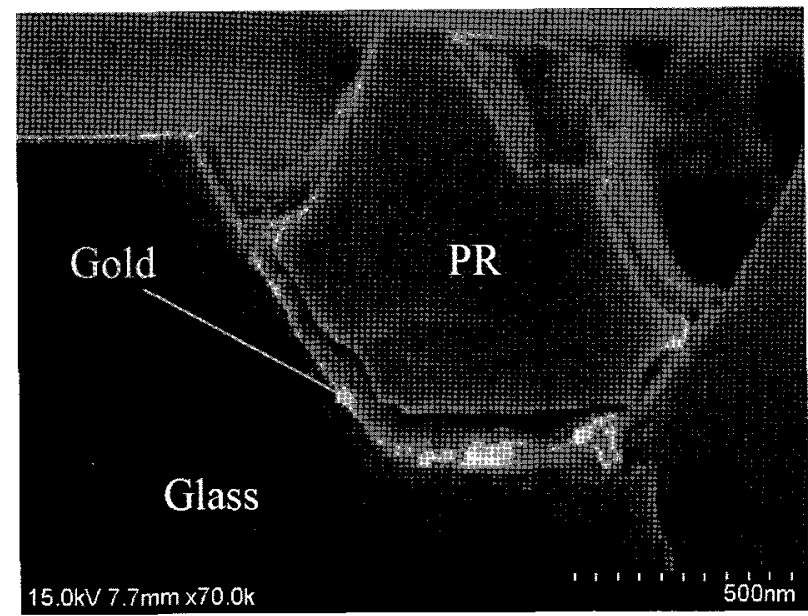

Fig. 8. Cross sectional view after additional UV flood exposure from the backside and gold wet etching

posure and gold wet etching, where the gold layer inside the glass trench was used as a self-aligned photomask.

The PR was patterned as a reversal image of the gold pattern in the glass trench after backside UV flood exposure. The sidewall gold residues were effectively removed using gold wet etching and the PR was also damaged from etchant as shown in Fig. 8.

\section{Conclusion}

We have proposed a simple fabrication method for nano-scale metal lines inside glass trenches. The structure was built for the application of nano-scale beam resonators. Self alignment with flood exposures from the backside effectively eliminated the problems of alignments between layers, which especially becomes significant in nano-scale devices. The PR developed during the flood exposure from the backside was highly affected by the sidewall slopes of the glass trenches. Two kinds of equipment for dry etching of glass were compared to obtain the relationship between the slopes of the glass trenches and the developed PR. The Precison-5000 has shown a glass trench profile of $54.0^{\circ}$ which successfully led to a TDMR-AR-87 slope of $63.5^{\circ}$ by flood exposure from the backside. The low slope of the PR was effective during the metal evaporation and lift-off process inside the glass trench. The metal residues left behind after the lift-off process can be removed by another flood exposure from the backside and wet etching. The self alignment and flood exposures have proven that the fabrication of nano-scale structures is possible without alignment related drawbacks. Further developments with overhanging plates will realize nano-scale vertical resonators. 


\section{References}

[1] K. L. Ekinci, and M. L. Roukes, "Nanoelectromechanical systems," Rev. Sci. Instrum., vol. 76, 061 101, 2005.

[2] A. N. Cleland, and M. L. Roukes, "Monocrystalline silicon carbide nanoelectromechanical systems," Appl. Phys. Lett. 78, 162, 2001.

[3] K. L. Ekinci, X. M. H. Huang, and M. L. Roukes, "Ultrasensitive nanoelectromechanical mass detection," Appl. Phys. Lett. 84, 4469, 2004.

[4] Ce'dric Durand, Fabrice Casset, Pascal Ancey et al, "Silicon on nothing MEMS electromechanical resonator," Microsystem Technologies, vol. 14, issue 7, pp. 1027-1033, 2008.

[5] Marc Madou, Fundamentals of MICROFABRICATION. CRC press, Chapter 1, 1997.

[6] Young-Soo Sohn, Moon-Gyu Sung, Young-Mee Lee et al, "Photoresist Exposure Paremeter from Refractive Index Change during Exposure," Jpn. J. Appl. Phys. vol. 37, pp. 6877-6883, 1998.

[7] Pil S. Hong, Jisung Kim, and Hong H. Lee, "Contrast modified room-temperature imprint lithography," Appl. Phys. Lett. 88, 173105, 2006.

[8] Shijic Liu, Jianyong Ma, and Yunxian Jin et al, "Optimization of thin-film design for multi-layer dielectric grating," Applied Surface Science, vol. 253, issue 7, pp. 3642-3648, 2007.

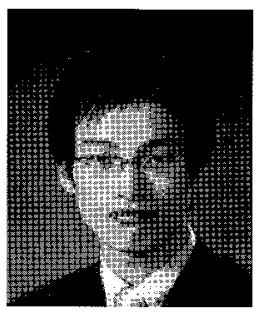

Yong-Seok Lee received his B.S. degree from the School of Electrical and Electronics Engineering at Chung-Ang University, Seoul, Korea, in 2007, his M.S. degree from the School of Electrical Engineering and Computer Science at Seoul National University, Seoul, Korea, in 2009, and is currently working towards his Ph.D. degree at the School of Electrical Engineering and Computer Science, Seoul, Korea, at Seoul National University. He is currently with the RF MEMS group, Micro Sensors and Actuators (MiSA) Laboratory, Seoul National University. His research interests are RF MEMS resonators, RF MEMS switches, and RF devices using MEMS technology.

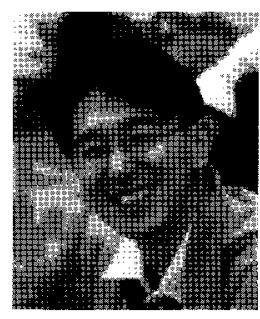

Yun-Ho Jang received his B.S., M.S. and Ph.D. degrees from the School of Electrical Engineering and Computer Science at Seoul National University, Seoul, Korea, in 1999, 2001, and 2005, respectively. His doctoral dissertation concerned the robust modeling, fabrication and experiment of a silicon micromirror array and its application in bio-molecular synthesis. In 2005, he joined Samsung Electronics, Co. Ltd., Yongin, Korea, as a senior researcher, where he participated in the development of a CMOS image sensor, particularly in small pixel and high resolution active pixels. In 2008, he joined Seoul National University, where he is currently a research professor of the School of Electrical Engineering and Computer Science. His current research interests are focused on the modeling, design, fabrication and testing of optical and RF MEMS devices.

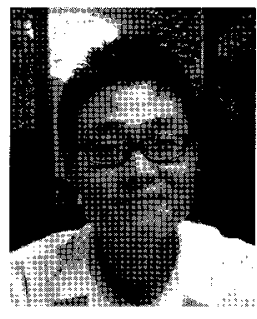

Yong-Seung Bang received his B.S. and M.S. degrees from the School of Electrical Engineering and Computer Science at Seoul National University, Seoul, Korea, in 2005 and 2007, respectively, and is currently working towards his Ph.D. degree at the School of Electrical Engineering and Computer Science at Seoul National University, Seoul, Korea. He is currently with the RF MEMS group, Micro Sensors and Actuators (MiSA) Laboratory, Seoul National University. His research interests are RF MEMS probe systems for cancer detection and treatment, MEMS packaging, RF MEMS switches, and RF devices using MEMS technology.

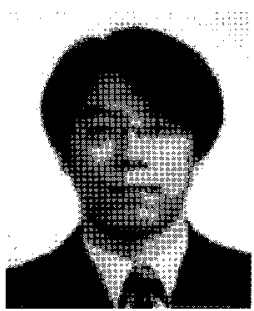

Jung-Mu Kim was born in Jeonju, Korea, in 1977. He received his B.S. degree in Electrical Engineering from Ajou University, Suwon, Korea, in 2000, his M.S. and Ph.D. degrees from the School of Electrical Engineering and Computer Science at Seoul National University, Seoul, Korea, in 2002 and 2007, respectively. From 2007 to 2008 , he was with the University of California at San Diego as a post doctoral researcher, where he was involved in the reliability of MEMS switches and MEMS filters. He then joined the faculty of the Division of Electrical, Electronic and Computer Engineering, Chonbuk National University. His current research activities include the design of RF MEMS, optical MEMS, micro actuators, and micromachined devices for the biological measurements system. 


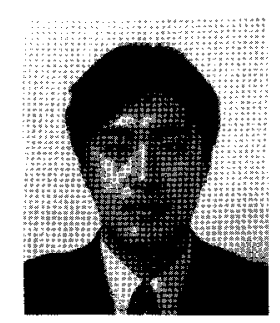

Jong-Man Kim received his B.S. degree from the School of Electrical and Electronics Engineering at Chung-Ang University in 2002, his M.S. and Ph.D. degrees from the School of Electrical Engineering and Computer Science at Seoul National University in 2004 and 2007, respectively. From 2007 to 2008, he was with the School of Chemical and Biological Engineering, Seoul National University, as a post doctoral researcher. In 2008, he joined the faculty of the College of Nano Science and Technology, Pusan National University. His current research interests are focused on the design, fabrication and characterization of MEMS/NEMS-based sensors and actuators including RF MEMS devices, bio-chips, chemical sensors, etc.

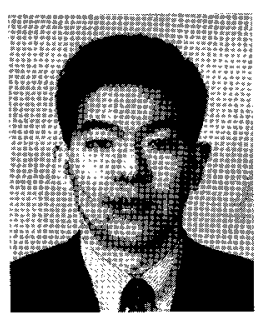

\section{Yong-Kweon Kim}

He received the B.S. and M.S. degrees in electrical engineering from Seoul National University, Seoul, Korea, in 1983 and 1985, respectively, and the Dr. Eng. Degree from the University of Tokyo, Tokyo, Japan, in 1990. His doctoral dissertation concerned modeling, design, fabrication, and testing of microlinear actuators in magnetic levitation using high critical temperature superconductors. In 1990, he joined the Central Research Laboratory, Hitachi Ltd., Tokyo, Japan, where he was a Researcher involved with actuators of hard disk drives. In 1992, he joined Seoul National University, where he is currently a Professor with the School of Electrical Engineering and Computer Science. His current research interests are modeling, design, and fabrication and testing of MEMS and their applications, especially inertial measurement units (IMUs), RF, optics, and biotechnology 